\title{
Counterpoint: Routine Daily Physical Exams Add Value for the Hospitalist and Patient
}

Laura C McNamara, $\mathrm{MD}^{1,2}$, and Zahir Kanjee, MD, MPH ${ }^{1,2 \star}$

${ }^{1}$ Beth Israel Deaconess Medical Center, Boston, Massachusetts; ${ }^{2}$ Harvard Medical School, Boston, Massachusetts.

W e read with interest the perspective of Drs Rodman and Warnock, ${ }^{1}$ but disagree with the authors on several points. We find that the routine daily physical examination, often neglected or unappreciated amidst technological advances and new diagnostic testing, provides significant value to both hospitalist and patient in terms of diagnosis, treatment, patient-centered care, and maintaining the patient-doctor relationship.

First, the daily physical exam provides the practice that hospitalists need to develop and maintain necessary diagnostic finesse. We are taught fundamental physical exam maneuvers in medical school, but these skills often atrophy during residency and throughout our careers. ${ }^{2}$ This leaves us, as practicing physicians, potentially worse at these competencies than when we were students. The daily physical exam, on the other hand, provides frequent and effective practice to keep up and build upon these skills. We gain in part from our repeated normal exams, which help us to skillfully recognize the rare abnormal finding; a hospitalist must likely feel hundreds of normal abdomens to reliably discover a furtive abdominal mass. Our exams also benefit from several forms of prompt and relevant feedback, including that which is provided by subspecialist consultants (like a cardiologist agreeing with your assessment of jugular venous pressure) and other diagnostic tests (like the echocardiogram without the valvulopathy thought to be detected at the bedside). The physical examination is best learned at the bedside, and the daily exam offers an unparalleled opportunity to do so.

Such continual skill improvement is necessary for hospitalists to accurately apply data from the evidence-based physical diagnosis literature. Many studies of the utility of various physical exam findings ${ }^{3}$ involve maneuvers performed by experts; to truly apply their results to our generalist practice, we are required to push ourselves to obtain the diagnostic expertise of the specialist. The daily physical examination, being the most concrete way for hospitalists to do so, is therefore essential to practicing better evidence-based physical diagnosis.

Beyond these larger benefits of the daily physical examination on our own practice and skills, patients in our care benefit

*Corresponding Author: Zahir Kanjee, MD, MPH; Email: zkanjee@ bidmc.harvard.edu; Telephone: 617-754-4677; Twitter: @zahirkanjee. Published online first August 18, 2021.

Received: March 13, 2021; Revised: June 19, 2021; Accepted: June 23, 2021

๑ 2021 Society of Hospital Medicine DOI 10.12788/jhm.3671 diagnostically from these exams as well. Time and again, we see an inadequate or incomplete physical exam leading to errors or adverse patient outcomes. ${ }^{4,5}$ Even after completion of initial laboratory and imaging tests, laying of hands and stethoscope can lead to dramatic changes in inpatient diagnosis and management. ${ }^{6}$ The subsequent routine daily physical provides fresh opportunities to reexamine the evidence for or against our own working diagnoses and management plans. The adage that "you don't know what you don't know" is especially fitting here. We often do not know to look for and rule a disease process in or out if it is not on our initial differential on hospital day one or two; the daily physical exam allows us to be on the lookout for diagnoses we have not yet considered. The two of us have more than once made a serendipitous discovery of a new rash or other physical finding on hospital day three or four that helps suggest another, and ultimately correct, final diagnosis. Particularly in a setting in which so many inpatient diagnoses are wrong and can lead to patient harm, the daily physical examination provides an important check on our own diagnostic reasoning.

Even if we are right about the diagnosis, the daily exam also allows for timely recognition of complications from our management. Listening to each patient's lungs every day, including those of patients with seemingly unrelated lower-extremity cellulitis, means we will more promptly notice when they retain fluid due to as yet unknown underlying heart failure. Those subtle bibasilar crackles not only become diagnostically useful, but also allow us the possibility of intervening and changing course even before the patient reports shortness of breath or the nurse notes hypoxemia on routine vital signs a day or two later. In an era when our treatment regimens are more complex, with frequent off-target results and side effects, the daily exam is a key screening tool for adverse outcomes in an increasingly ill population. Having a frequently updated and accurate baseline exam is also exceptionally important in the event of sudden neurologic deficits; an inpatient with new facial droop and left-arm weakness at 10 Am has much better treatment options if their hospitalist has conducted a routine basic neurologic exam that morning and can confidently provide a time when they were "last known well."

Finally, the daily physical examination is important to patientcentered care and potentially preventing physician burnout. ${ }^{8} \mathrm{~Pa}-$ tients have more confidence in us when we conduct a thorough exam. The ritual of the physical exam is also an important contributor to the patient-doctor relationship, and a daily exam can help strengthen that bond each morning. ${ }^{9}$ Such benefits 
also extend to physicians. Hospitalists are spending less and less time at the bedside, ${ }^{10}$ a reality at least partially responsible for rising rates of burnout. ${ }^{11}$ We all went into clinical medicine to take care of and connect with people. The daily physical examination offers valuable time to show our patients we care about them while also giving us the opportunity to spend time with them, rather than with the "iPatient" that can otherwise become our focus. ${ }^{12}$ In this way, the daily physical examination can be immensely satisfying and may not only inoculate against burnout but also contribute to a stronger patient-doctor relationship.

For so many reasons, the daily physical exam is of great benefit to hospitalists looking to develop and maintain diagnostic skills, to our patients as we stay on the lookout for unexpected diagnoses and complications, and to the relationships we have with those for whom we care. It is a practice worth not only continuing but celebrating.

Disclosures: The authors reported no conflicts of interest.

\section{References}

1. Rodman A, Warnock S. Point: routine daily physical exams in hospitalized patients are a waste of time. J Hosp Med. 2021;16(9):568-569. https:// doi.org/10.12788/jhm.3670
2. Vukanovic-Criley JM, Criley S, Warde CM, et al. Competency in cardiac examination skills in medical students, trainees, physicians, and faculty: a multicenter study. Arch Intern Med. 2006;166(6):610-616. https://doi.org/10.1001/ archinte.166.6.610

3. McGee S. Evidence-Based Physical Diagnosis. 4th ed. Elsevier; 2018.

4. Verghese A, Charlton B, Kassirer JP, Ramsey M, loannidis JPA. Inadequacies of physical examination as a cause of medical errors and adverse events: a collection of vignettes. Am J Med. 2015;128(12):1322-1324.e3. https:// doi.org/10.1016/j.amjmed.2015.06.004

5. Singh H, Giardina TD, Meyer AND, Forjuoh SN, Reis MD, Thomas EJ. Types and origins of diagnostic errors in primary care settings. JAMA Intern Med. 2013;173(6):418-425. https://doi.org/10.1001/jamainternmed.2013.2777

6. Reilly BM. Physical examination in the care of medical inpatients: an observational study. Lancet. 2003;362(9390):1100-1105. https://doi.org/10.1016/ S0140-6736(03)14464-9

7. Gunderson CG, Bilan VP, Holleck JL, et al. Prevalence of harmful diagnostic errors in hospitalised adults: a systematic review and meta-analysis. $B M J$ Qual Saf. 2020;29(12):1008-1018. https://doi.org/10.1136/bmjqs-2019-010822

8. Silverman B, Gertz A. Present role of the precordial examination in patient care. Am J Cardiol. 2015;115(2):253-255. https://doi.org/10.1016/j.amjcard.2014.10.031

9. Costanzo C, Verghese A. The physical examination as ritual: social sciences and embodiment in the context of the physical examination. Med Clin North Am. 2018;102(3):425-431. https://doi.org/10.1016/j.mena.2017.12.004

10. Malkenson D, Siegal EM, Leff JA, Weber R, Struck R. Comparing academic and community-based hospitalists. J Hosp Med. 2010;5(6):349-352. https:// doi.org/10.1002/jhm.793

11. Hipp DM, Rialon KL, Nevel K, Kothari AN, Jardine LDA. "Back to bedside": Residents' and fellows' perspectives on finding meaning in work. J Grad Med Educ. 2017;9(2):269-273. https://doi.org/10.4300/JGME-D-17-00136.1

12. Verghese A. Culture shock--patient as icon, icon as patient. N Engl J Med. 2008;359(26):2748-2751. https://doi.org/10.1056/NEJMp0807461

\section{Journal of Hospital Medicine}

\section{journalofhospitalmedicine.com}
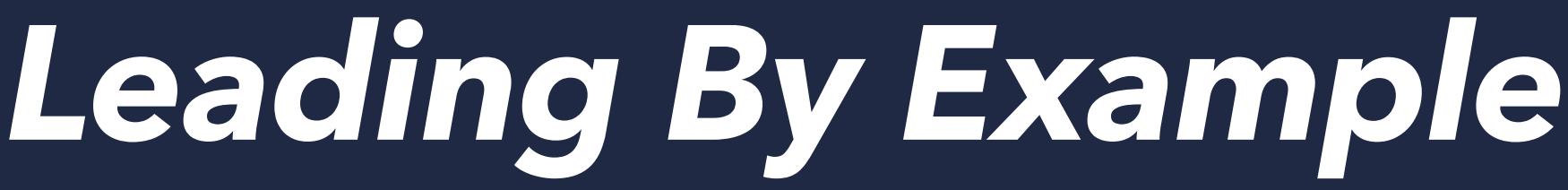

\section{\#HowWeHospitalist}

